\title{
Refractory Hypotension Caused By Prone Position in a Child Undergoing Scoliosis Corrective Surgery
}

\author{
Ye $\mathrm{F}^{1}$, Yang $\mathrm{J}^{2}$, Li $\mathrm{Q}^{1}$, Liu $\mathrm{W}^{1}$, Dou $\mathrm{Y}^{1}$, Huang $\mathrm{Z}^{2}$, Huang $\mathrm{W}^{1}$ and Shu $\mathrm{H}^{* 1,3}$ \\ ${ }^{1}$ Department of Anesthesiology, The First Affiliated Hospital of Sun Yat-sen University, Guangzhou, China \\ ${ }^{2}$ Department of Spine Surgery, The First Affiliated Hospital of Sun Yat-sen University, Guangzhou, China \\ ${ }^{3}$ Department of Anesthesiology, Guangdong Second Provincial People's Hospital, 466\# Xingang road, \\ Guangzhou, Guangdong, 510317, China
}

${ }^{*}$ Corresponding author: Shu H, Department of Anesthesiology, Guangdong Second Provincial People's Hospital, Guangzhou, Guangdong, China, Fax: +86-20-87330736, Tel: +86-20-87755766-8273, E-mail: shuhaihua@hotmail.com

Citation: Ye F, Yang J, Li Q, Liu W, Dou Y, et al. (2014) Refractory Hypotension Caused By Prone Position in a Child Undergoing Scoliosis Corrective Surgery. J Case Rep Stud 2(5): 505. doi: 10.15744/2348-9820.2.205

Received Date: September 06, 2014 Accepted Date: October 07, 2014 Published Date: October 10, 2014

\begin{abstract}
We report a case of a teenager with scoliosis and pectus excavatum who developed intraoperative refractory hypotension associated with prone position during posterior surgical correction of scoliosis. In this case, it was more difficult for the anesthetists to figure out the reason leading to severe hypotension when a surgery was partially completed, because there might be many confounding factors during surgery. Furthermore, TEE was difficult to be considered to be first diagnosis choice when patient have already underwent posterior spinal fusion surgery, compared to the cases previous reported that the surgery still did not begin. Nevertheless, we managed to complete our partially completed surgery in a lateral prone position by one stage in a creative way. This report provides sufficient and logical differential diagnosis for the management of intraoperative hypotension associated with prone position. Moreover, our case strongly suggests that more detailed preoperative assessments are necessary to identify the potential risk of prone position on intraoperative blood pressure in scoliosis patients with pectus excavatum or Marfan's-like feature.
\end{abstract}

\section{Introduction}

Scoliosis is a complicated spinal deformity involving lateral curvature of the spine, vertebral body rotation, and angulation of the ribs. Posterior spinal fusion with instrumentation is a common treatment for scoliosis patients [1]. We report a case of a teenager with scoliosis and pectus excavatum who developed intraoperative refractory hypotension associated with prone position during posterior surgical correction of scoliosis.

\section{Observation}

A 16-year-old male (weight $48 \mathrm{~kg}$ ) with severe scoliosis presented complaining of marked lumbar back pain and deformity of spine for one year duration. He denied any preexisting antecedent illness and past medical history. On physical examination, he had apparent scoliosis with right rib hump, pectus excavatum and winged deformity of left shoulder (Figure 1A and B). In addition, he had mild heaving axillary pulses, slender limbs, arachnodactylia and arthrochalasis (Figure 1A and B). The neurologic examination was unremarkable. The preoperative electrocardiogram (ECG) indicated a mild ST-segment elevation but the preoperative echocardiography indicated normal structure and systolic function. The preoperative radiograph revealed a cobb angle of $79^{\circ}$ lumbar scoliosis and $23^{\circ}$ lumber kyphosis. His ventilation function was mildly depressed, while the lung capacity was enhanced, both of which were not severe. The clinical laboratory examination results were unremarkable. Given all of these, no specific diagnosis of Marfan's syndrome was made for the patient and he was diagnosed as idiopathic scoliosis.

A surgery of posterior spinal fusion with a combination of rods and pedicle screws was suggested to patient. According to the surgical procedure, a combined intravenous and inhalation anesthesia was chosen. The informed consent was obtained from the patient and his parents. Anesthesia was induced with midazolam, propofol and fentanyl. Endotracheal intubation was facilitated by cis-atracurium. After anesthesia induction, the arterial line and right internal jugular vein line were placed. Anesthesia was maintained with sevoflurane at $1-1.5 \%$, propofol at $1-2 \mathrm{mcg} / \mathrm{kg} / \mathrm{min}$, remifentanil at $0.2 \mu \mathrm{g} / \mathrm{kg} / \mathrm{min}$. Heart rate (HR), invasive blood pressure (BP), central venous pressure (CVP), $\mathrm{SpO}_{2}, \mathrm{EtCO}_{2}$ and esophageal temperature were monitored. Arterial blood gas was measured when it was necessary. Patient was placed in prone position with bolsters across his chest and hips (Figure $2 \mathrm{~A}$ ). During the early $70 \mathrm{~min}$ of surgery when patient was underwent incision, isolation and exposure and his vital signs were stable. With placement of pedicle screws, the patient's BP declined from 105/65 to 60/30 $\mathrm{mmHg}$ progressively, HR increased from $70-80$ to $100-110 \mathrm{bpm}$ with sinus rhythm, CVP increased from 7-8 to 9-11 cm $\mathrm{H}_{2} \mathrm{O} .5 \mathrm{mg}$ of ephedrine was twice administrated and 300 $\mathrm{ml}$ of hydroxyethyl starch was rapidly infused. The BP increased to $80-90 / 40-50 \mathrm{mmHg}$ and maintained no more than $10 \mathrm{~min}$. 
The BP deceased again to 50-60/30-40mmHg, HR kept increasing to $120-130 \mathrm{bpm}$, CVP kept to be 9-11 cm $\mathrm{H}_{2} \mathrm{O}$. A request for suspension of the surgery secondary to concerns of persistent hypotension was made. Blood loss (including volume of suction and from gauze) was assessed to be about $200 \mathrm{ml}$. Urine drainage was checked. The volume of urine was about $600 \mathrm{ml}$ and the color was limpid. Blood gas was measured immediately. It was found that the hemocrit was 35\%, similar to baseline (38\%) which was measured immediately after set up of arterial line. Partial pressure of oxygen was $398 \mathrm{mmHg}$. The plasma $\mathrm{pH}$ was 7.37, sodium was $138 \mathrm{mmol} / \mathrm{L}$, potassium was $3.8 \mathrm{mmol} / \mathrm{L}$, calcium was $1.19 \mathrm{mmol} / \mathrm{L}$, and glucose was $6.7 \mathrm{mmol} / \mathrm{L}$. Esophageal temperature increased slightly from $36.8^{\circ} \mathrm{C}$ to $37.4^{\circ} \mathrm{C}$. The $\mathrm{EtCO}_{2}$ rose from 37 to $40 \mathrm{mmHg}$. Skin assessment found no discernible. Tracheal tube was checked to be normal and found no hemorrhagic secretion. ECG was checked and found no significant change of ST segment and T wave. Massive hemorrhage, malignant hyperthermia and pulmonary embolism were excluded according to the above clinical data. Even no rash and redness on skin, the allergy shock was not easy to be excluded. Because of high CVP and tachycardia, cardiac failure may be the reason. Although ECG demonstrated no significant changes, myocardial infarction was not easy to be excluded. Thereby, methylprednisolone, lanatoside C, dopamine, epinephrine and norepinephrine were tried, respectively or combinedly, to improve the hemodynamic status of the patient but the effect was limited. When epinephrine and norepinephrine was continuously infused, the BP can be maintained at 70-90/40-60 mmHg, while the HR increased to be 140 $150 \mathrm{bpm}$ and CVP to be $14-18 \mathrm{~cm} \mathrm{H} \mathrm{H}_{2} \mathrm{O}$. We had to stop the surgery to improve the hemodynamic condition. The skin was closed and the patient was turned over. Immediately after the patient was turned to supine position, his vital signs changed to be normal rapidly. The BP increased to $130-150 / 80-90 \mathrm{mmHg}$, $\mathrm{HR}$ decreased to $90-100 \mathrm{bpm}$ and the $\mathrm{CVP}$ decreased to 5-7 $\mathrm{cm}_{2} \mathrm{O}$. The vital signs remained stable even if the cardiovasoactive drugs were withdrawn. Until now the cause of hypotension was determined, which was the compression of the thoracic cavity by prone position. When we tried to compress the thoracic cavity by hands, the hypotension could be induced again. Because the surgery was half-finished, we tried to continue the surgery by placing the patient in a lateral prone position and putting longitudinal bolsters situated along right side of his body rather than across his chest and hips, for reducing the compression on thoracic cavity (Figure $2 \mathrm{~B}$ and $\mathrm{C}$ ). As expected, we finished the scheduled operation with stable vital signs. There were no complications during and after the operation.

\section{Discussion}

Hypotension is a common complication during operation. Usually, it may be caused by over-dose anesthetics, hypovolemia, hemorrhage, allergy, cardiac failure, or myocardial infarction. And rarely, it may be caused by pulmonary embolism or malignant hyperthermia. In this case, a child with scoliosis and pectus excavatum appeared an intraoperative refractory hypotension during a posterior spinal corrective surgery. It was a rare case that patient position caused such a severe hypotension in a spinal corrective surgery. To the best of our knowledge, only two reports discussed the situation by Bafus et al and Alexianu et al [2,3]. In their case, the patient's blood pressure decreased soon after they placed the patient in prone position. The hypotension was no response to routine treatment. The surgery was then stopped. Bafus et al performed a transesophageal echocardiogram (TEE) to evaluate the heart and aortic root in supine and prone positions and found pectus excavatum was culprit. They corrected the pectus excavatum at first and carried on the spinal fusion surgery subsequently with stable hemodynamic status [2]. Alexianu et al determined the causes by the subsequent MRI and transthoracic echocardiography and completed the surgery by careful intraoperative management such as TEE monitoring [3]. In our case, however, the patient's blood pressure was stable at the early 70 min therefore the surgery was proceeded. It led our case to be special: firstly, it was more difficult to determine the reason leading to hypotension during surgery because 1) there might exist confounding factors if surgery was underwent, as described above 2) TEE was difficult to be considered to be first diagnosis choice when patient underwent posterior spinal fusion surgery; secondly, we managed to complete our half-finished surgery in a lateral prone position (Figure $2 \mathrm{~B}$ and $\mathrm{C}$ ).

It was reported that the prone position resulted in a significant reduction of left ventricular end-diastolic volume, stroke volume, and the increase of sympathetic nerve activity [4]. But the hemodynamic changes caused by prone position should be limited as the mean arterial pressure could be maintained by increased systemic vascular resistance, as it manifested in the early 70 min in this case $[4,5]$. Why did the hypotension progress so severe when the surgeons began to implant the pedicle screws? The reason was unclear. However, it was found, in patient with scoliosis and pectus excavatum, that the inflow gradient would increase to $8 \mathrm{~mm} \mathrm{Hg}$ (peak 17) with an E:A wave reversal when placing the patient in prone position with the bolsters on the Jackson table [2]. It suggested that the prone position may bring about severe hemodynamic changes in some special patients. In this case, the child was actually a Marfan's syndrome-like patient as supported by some signs, such as slender limbs, mild heaving axe pulses, arachnodactylia, arthrochalasis, pectus excavatum and scoliosis, even a specific diagnosis of Marfan's syndrome was difficult to make. Marfan's syndrome is a systemic disorder of connective tissue caused by mutations in the extracellular matrix protein fibrillin 1, and its manifestations are typically involved with the cardiovascular, skeletal, and ocular systems [6]. It is reported that about 71 percents of patients with Marfan's syndrome have scoliosis [7]. With the Marfan's-like feature, the patient manifested a significant deformation (e.g. pectus excavatum) and softening of thoracic cavity, which makes it easy to be compressed. In the early $70 \mathrm{~min}$ when the surgery was in stage of exposure, the compression on the thoracic cavity was still tolerable. However, in the next stage of surgery (implanting the pedicle screws) the compression was under decompensation. The compression on the thoracic cavity and heart led to a significant limitation of inflow and diastole of heart, therefore, caused severe hypotension, tachycardia and high CVP. 

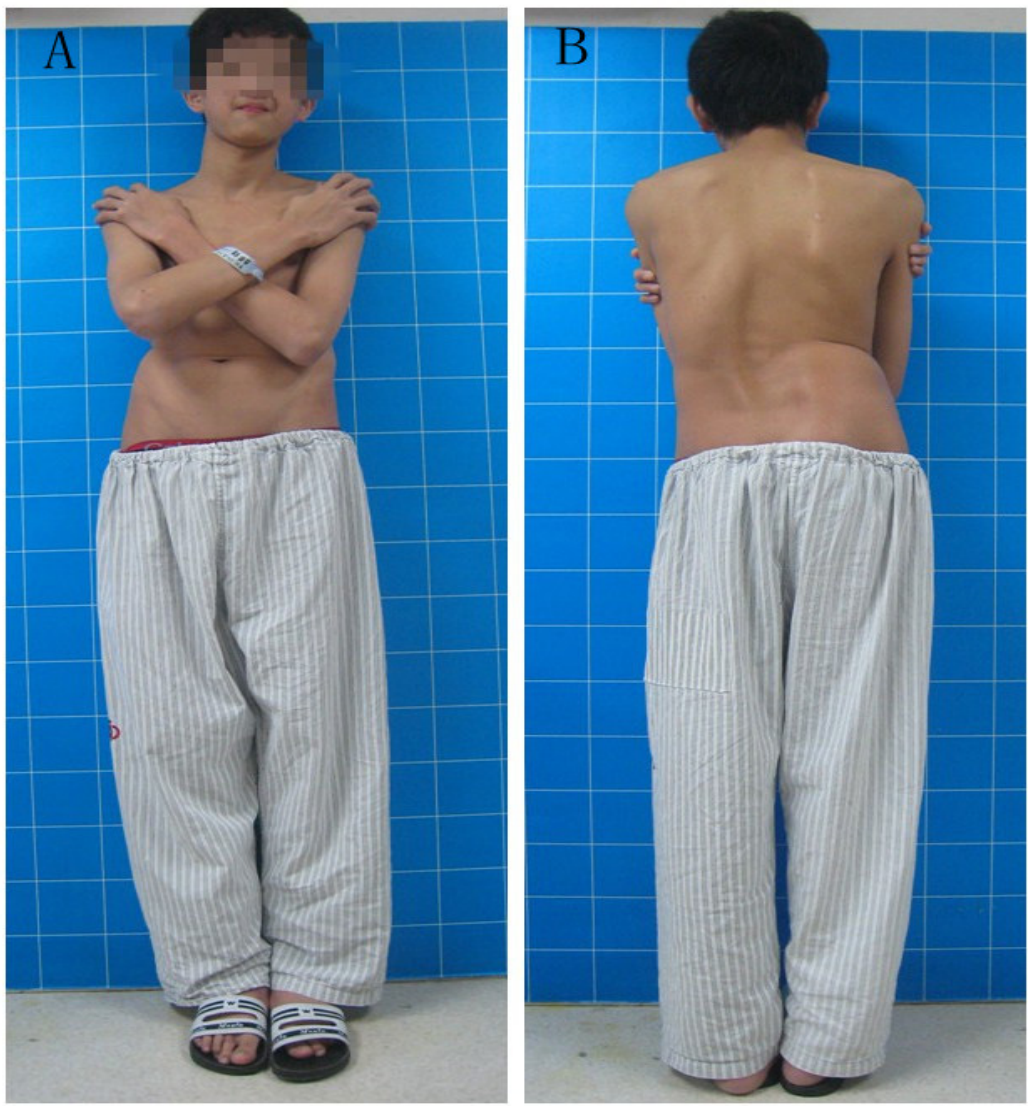

Figure 1: Preoperative view of the patient. A - Frontal view; B - Posterior view
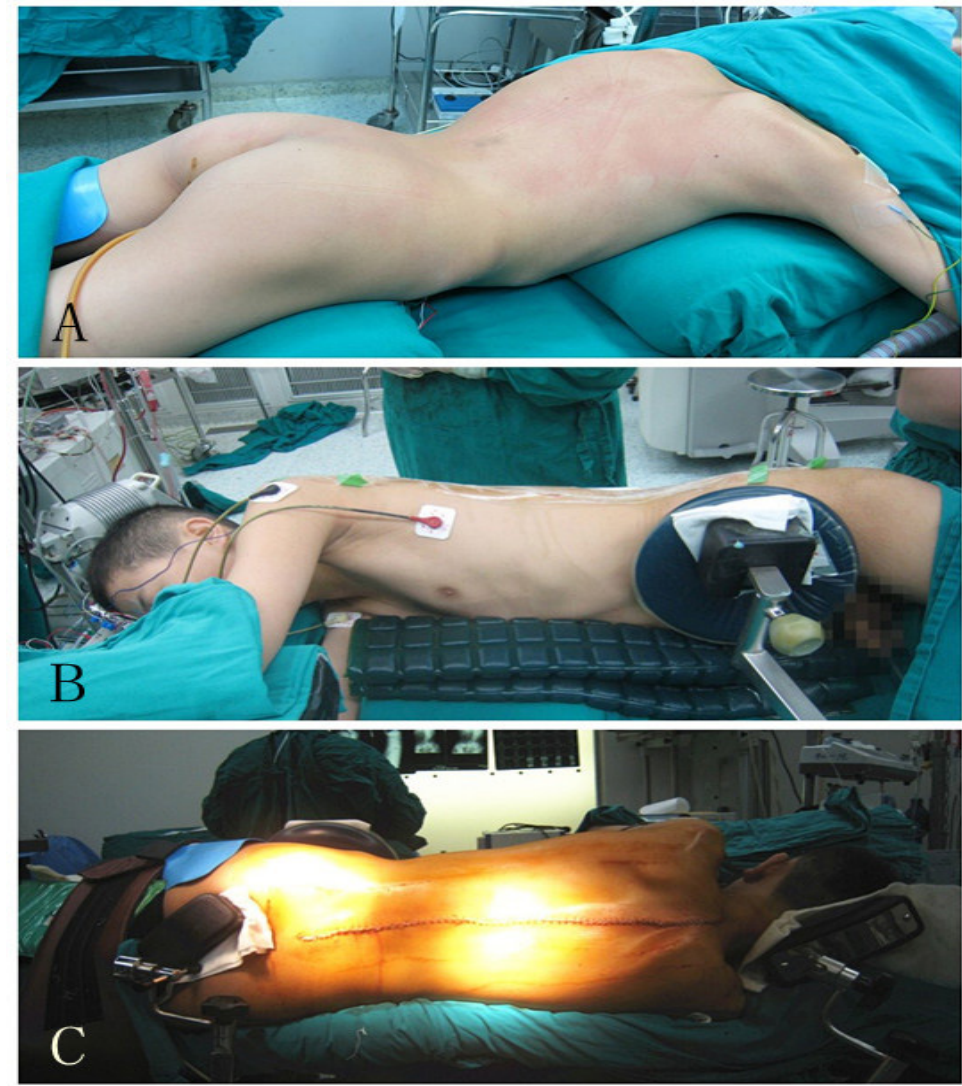

Figure 2: Intraoperative positions of the patient. A - Routine position: the patient was placed in prone position with bolsters across his chest and hips; B\&C - Corrected position: The patient was placed in a lateral prone position and with longitudinal bolsters situated along right side of his body (B - frontal view, $\mathbf{C}$ - posterior view) 
Our case strongly suggests that a more detailed preoperative assessment is necessary for understanding the potential risk in scoliosis patients with pectus excavatum or Marfan's-like feature. For example, making a preoperative prone position test or a thorax compression test to see if it induces changes of hemodynamic status; or making echocardiography in both spine and prone positions to evaluate the changes of heart and aortic root. The application of echocardiography through esophagus or thoracic wall provides the capacity to obtain detailed information about structure and function of the heart and great vessels [8,9]. If positive results appear, a planned intervention should be carried out. For example, correcting the deformation of thoracic cavity before spinal fusion surgery [2], providing intraoperative TEE monitor to obtain more useful intraoperative hemodynamic parameters, or reducing thoracic pressure [3]. For us, a lateral prone position with longitudinal bolsters situated along body side was taken to reduce the pressure on thoracic cavity and heart.

\section{Conclusion}

This report provides sufficient and logical differential diagnosis and therapeutic measures of perioperative hypotension caused by patient position. Moreover, our case strongly suggests that a more detailed preoperative assessment is necessary for understanding the potential risk in scoliosis patients with pectus excavatum or Marfan's-like feature.

\section{References}

1. Janicki JA, Alman B (2007) Scoliosis: Review of diagnosis and treatment. Paediatr Child Health 12: 771-6.

2. Bafus BT, Chiravuri D, van der Velde ME, Chu BI, Hirshl R, et al. (2008) Severe hypotension associated with the prone position in a child with scoliosis and pectus excavatum undergoing posterior spinal fusion. J Spinal Disord Tech 21: 451-4.

3. Alexianu D, Skolnick ET, Pinto AC, Ohkawa S, Roye DJ, et al. (2004) Severe hypotension in the prone position in a child with neurofibromatosis, scoliosis and pectus excavatum presenting for posterior spinal fusion. Anesth Analg 98: 334-5.

4. Schaefer WM, Lipke CS, Kuhl HP, Koch KC, Kaiser HJ, et al. (2004) Prone versus supine patient positioning during gated 99mTc-sestamibi SPECT: effect on left ventricular volumes, ejection fraction, and heart rate. J Nucl Med 45: 2016-20.

5. Edgcombe H, Carter K, Yarrow S (2008) Anaesthesia in the prone position. Br J Anaesth 100: 165-83.

6. Yang XS, Sun JP, Felner JM. (2010) Marfan's Syndrome. 152-3.

7. Robins PR, Moe JH, Winter RB (1975) Scoliosis in Marfan's syndrome. Its characteristics and results of treatment in thirty-five patients. J Bone Joint Surg Am 57: 358-68.

8. Cameli M, Lisi M, Righini FM, Mondillo S (2012) Novel echocardiographic techniques to assess left atrial size, anatomy and function. Cardiovasc Ultrasound 10: 4 .

9. Roman MJ, Devereux RB, Kramer-Fox R, O’ Loughlin J (1989) Two-dimensional echocardiographic aortic root dimensions in normal children and adults. Am J Cardiol 64: 507-12. 